\title{
SYNCHRONIZATION VIA PINNING CONTROL ON GENERAL COMPLEX NETWORKS*
}

\author{
WENWU YUं, GUANRONG CHEN ${ }^{\ddagger}$, JINHU LÜ§, AND JÜRGEN KURTHS
}

\begin{abstract}
This paper studies synchronization via pinning control on general complex dynamical networks, such as strongly connected networks, networks with a directed spanning tree, weakly connected networks, and directed forests. A criterion for ensuring network synchronization on strongly connected networks is given. It is found that the vertices with very small in-degrees should be pinned first. In addition, it is shown that the original condition with controllers can be reformulated such that it does not depend on the form of the chosen controllers, which implies that the vertices with very large out-degrees may be pinned. Then, a criterion for achieving synchronization on networks with a directed spanning tree, which can be composed of many strongly connected components, is derived. It is found that the strongly connected components with very few connections from other components should be controlled and the components with many connections from other components can achieve synchronization even without controls. Moreover, a simple but effective pinning algorithm for reaching synchronization on a general complex dynamical network is proposed. Finally, some simulation examples are given to verify the proposed pinning scheme.
\end{abstract}

Key words. complex network, pinning control, synchronization, strongly connected network, directed spanning tree, weakly connected network, directed forest

AMS subject classifications. 93C10, 68M12, 93B25, 93A15

DOI. $10.1137 / 100781699$

1. Introduction. Collective behaviors in complex networks and systems $[3,10$, $11,28,35,39]$ have attracted increasing attention in recent years due to their wide applications in physics, mathematics, engineering, biology, etc. Synchronization, similarly consensus, is a typical collective behavior in complex dynamical networks and systems. Since the pioneering work of Pecora and Carroll [24], chaos synchronization [2] has received a great deal of attention due to its potential applications in secure communications, biological neural networks [47], genetic regulatory networks [57], parameter estimation, and so on. In nature, there are large numbers of vertices in real-world complex networks, so it is of great interest to study network synchronization

*Received by the editors January 4, 2010; accepted for publication (in revised form) January 4, 2013; published electronically April 3, 2013. This work was supported by the National Natural Science Foundation of China under grants 61104145, 61025017, 11072254, and 61203148, the Natural Science Foundation of Jiangsu Province of China under grant BK2011581, the Research Fund for the Doctoral Program of Higher Education of China under grant 20110092120024, the Fundamental Research Funds for the Central Universities of China, the DAAD Scholarship (Deutscher Akademischer Austauschdienst Dienst) under Ref. 423, the Australian Research Council (ARC), Discovery Scheme under grant DP130104765, and the Hong Kong Research Grants Council under GRF grant CityU1109/12. A preliminary version of this paper appears in Proceedings of the 10th World Congress on Intelligent Control and Automation (WCICA), IEEE Press, Piscataway, NJ, 2012, pp. 1930-1935.

http://www.siam.org/journals/sicon/51-2/78169.html

$\dagger$ Department of Mathematics, Southeast University, Nanjing 210096, China (wenwuyu@gmail. com,wwyu@seu.edu.cn).

${ }^{\ddagger}$ Department of Electronic Engineering, City University of Hong Kong, Kowloon, Hong Kong (gchen@ee.cityu.edu.hk).

$\S$ The Key Laboratory of Systems and Control, Institute of Systems Science, Academy of Mathematics and Systems Science, Chinese Academy of Sciences, Beijing 100190, China (jhlu@iss.ac.cn).

IResearch Domain IV, Institute for Climate Impact Research, Potsdam 14473, Germany, and Institute for Complex Systems and Mathematical Biology, University of Aberdeen, Aberdeen AB24 3FX, UK (Juergen.Kurths@pik-potsdam.de).

1395 
$[1,8,18,19,36,37,48,49,56,58,60,61]$ and consensus $[6,51,52,53,55,59,64]$ in very large-scale complex networks. First, local synchronization, meaning that synchronization can be reached within a certain region, was studied by the transverse stability to the synchronization manifold [36, 37]. Further study about local synchronization in time-varying complex networks was also reported [19]. For the global behavior, a distance function from the collective states of the network vertices to the synchronization manifold was used $[40,44]$ to study global synchronization of coupled networks and systems $[18,48,49]$. By designing some adaptive laws on the weights and coupling strength, synchronization in adaptive networks was shown $[8,60,61]$.

In addition to the commonly studied undirected networks $[18,19,36,37,38,45$, $46,48,49,61,62]$, directed networks are also used to represent the interactive topology between the vertices. Actually, many real-world networks are directed, such as communication networks, the World Wide Web, email networks, and electrical power grids, to name just a few. Theoretically, the convergence analysis of synchronization for directed complex networks is more challenging than for undirected ones [41]. One contribution of this paper is that the convergence of a synchronization problem via pinning control on directed networks is theoretically analyzed, yielding some useful criteria for future applications.

In the case where the whole network can not synchronize by its intrinsic structure, some controllers may be designed and applied to drive the network to synchronization. However, it is literally impossible to add controllers to all vertices in a large-scale network. To reduce the number of controlled vertices, some local feedback injections may be applied to a fraction of network vertices, which is known as pinning control $[7,9,12,15,16,17,25,26,32,33,34,38,42,43,45,46,54,62,63]$. For example, in the leader-follower control or distributed tracking problem, a group of mobile agents can coordinate so that they can track the leader $[13,50,55]$. The key issue is to design the appropriate control input where only a small fraction of agents can measure the leader's information. The main objective in pinning control is to apply controllers on a fraction of all the vertices such that the whole network can reach synchronization. Earlier, pinning control of spatiotemporal chaos was studied on a coupled map lattice [12]. As a result of the broad studies about complex networks, both specific and random pinning schemes were applied on complex networks to achieve an equilibrium point $[16,38]$, where the specific pinning of the vertices with large degrees was shown to require a smaller number of controlled vertices than the random pinning scheme. Later, a virtual vertex was introduced in the network, and pinning controllability of complex networks could be transformed to synchronization of the augmented complex networks $[33,34]$. It was shown that if the coupling strength is sufficiently large, the network can achieve synchronization by pinning only one vertex in the network [7]. The pinning-controllability problem of the undirected complex networks was reformulated as a global asymptotic stability problem [25, 26], and some new criteria concerning the selection of pinned vertices and effects of network topology have been discussed. As for adaptive complex networks, it was found that a network under a typical framework can realize synchronization subject to any linear feedback pinning scheme by using adaptive tuning of the coupling strength [7, 54]. Some distributed adaptive laws based on the information of the neighbors were designed on the coupling strength of complex networks for reaching synchronization [17]. Interestingly, it was shown that the vertices with very small degrees should be pinned first when the coupling strength is small $[15,54]$ and that the derived pinning condition with controllers given in a high-dimensional matrix criterion can be reduced to a low-dimensional matrix criterion without any pinning controllers involved [54, 63]. 
Nowadays, it is still quite challenging to consider the pinning scheme on a general complex network. Recently, pinning synchronization on directed networks was discussed [32, 62]. However, the derived conditions were based on the sum of the Laplacian matrix and its transpose which may have some eigenvalues with negative real parts. Then, by choosing a standard Lyapunov function, the derivative of this function along the trajectory of the system might be positive such that the whole system can not converge. Therefore, these conditions lack the structural information of the network, and the key factor needed for reaching network synchronization is not revealed. By considering the control signal as the state of a virtual system, the authors of $[42,43]$ gave a general pinning scheme in directed networks based on network structure and algebraic graph.

The main contribution of this paper is that it further addresses the problem of pinning synchronization based on the more detailed structural information of general complex dynamical networks, particularly strongly connected networks, networks with a directed spanning tree, weakly connected networks, and directed forests. (A simplified version of pinning synchronization for complex networks with strongly connected topologies was reported in [56].) We offer some new findings. First, it is found that the vertices with very small in-degrees should be pinned first. Second, the condition for reaching synchronization with controllers is shown to be equivalent to a simple condition without control, which implies that the vertices with very large out-degrees should also be pinned. Third, a criterion for achieving synchronization on networks with a directed spanning tree, which can be composed of many strongly connected components, is derived. It is found that the components with very few connections coming from other components should be controlled, and the components with many connections coming from other components can achieve synchronization even without control. Finally, an effective pinning algorithm for reaching synchronization on a general complex dynamical network is proposed.

The rest of the paper is organized as follows. In section 2, some preliminaries about model formulation and graph theory are briefly outlined. Some pinning synchronization criteria on strongly connected networks, networks with a directed spanning tree, and general complex dynamical networks are given in sections $3-5$. In section 6 , the pinning scheme on a representative network is simulated to illustrate the theoretical analysis. Conclusions are drawn in section 7 .

2. Preliminaries. Consider a complex dynamical network consisting of $N$ identical vertices with linearly diffusive coupling $[18,19,36,37,44,49,61]$, described by

$$
\dot{x}_{i}(t)=f\left(x_{i}(t), t\right)+c \sum_{j=1, j \neq i}^{N} G_{i j} \Gamma\left(x_{j}(t)-x_{i}(t)\right), \quad i=1,2, \ldots, N,
$$

where $x_{i}(t)=\left(x_{i 1}(t), x_{i 2}(t), \ldots, x_{i n}(t)\right)^{T} \in \mathbb{R}^{n}$ is the state vector of the $i$ th vertex, $f: \mathbb{R}^{n} \times \mathbb{R}^{+} \longrightarrow \mathbb{R}^{n}$ is a continuously differentiable vector function, $c$ is the coupling strength, $\Gamma \in \mathbb{R}^{n \times n}$ is the inner coupling matrix, and $G=\left(G_{i j}\right)_{N \times N}$ is the coupling configuration matrix representing the topological structure of the network. $G_{i j}$ are defined as follows: if there exists a connection from vertex $j$ to vertex $i$, then $G_{i j}>0$; if there exists a connection from vertex $i$ to vertex $j$, then $G_{j i}>0$; otherwise, $G_{i j}=$ $0(j \neq i)$; and the diagonal elements of matrix $G$ are defined by

$$
G_{i i}=-\sum_{j=1, j \neq i}^{N} G_{i j}
$$


which ensures the diffusion $\sum_{j=1}^{N} G_{i j}=0$. Equivalently, due to the diffusion, network (2.1) can be rewritten in a simpler form as follows:

$$
\dot{x}_{i}(t)=f\left(x_{i}(t), t\right)+c \sum_{j=1}^{N} G_{i j} \Gamma x_{j}(t), \quad i=1,2, \ldots, N .
$$

Consider also a solution $s(t)$ of an isolated vertex, satisfying

$$
\dot{s}(t)=f(s(t), t) .
$$

Here, $s(t)$ may be an equilibrium point, a periodic orbit, or even a chaotic solution, which can be viewed as the state of the reference vertex, or as the virtual leader in multi-agent dynamical systems [23].

The pinning strategy is applied on a small fraction $\delta(0<\delta<1)$ of vertices in the network $[7,54,38]$. Suppose that the vertices $q_{1}, q_{2}, \ldots, q_{l}$ are selected to be pinned, where $l=\lfloor\delta N\rfloor$ represents the integer part of the real number $\delta N$. Then, the controlled complex network can be conveniently described by

$$
\begin{aligned}
& \dot{x}_{q_{i}}(t)=f\left(x_{q_{i}}(t), t\right)+c \sum_{j=1}^{N} G_{q_{i} j} \Gamma x_{j}(t)+u_{q_{i}}, \quad i=1,2, \ldots, l, \\
& \dot{x}_{q_{i}}(t)=f\left(x_{q_{i}}(t), t\right)+c \sum_{j=1}^{N} G_{q_{i} j} \Gamma x_{j}(t), \quad i=l+1, l+2, \ldots, N,
\end{aligned}
$$

where

$$
u_{q_{i}}(t)=-c d_{q_{i}} \Gamma\left(x_{q_{i}}(t)-s(t)\right) \in \mathbb{R}^{n}, \quad i=1,2, \ldots, l,
$$

are $n$-dimensional linear feedback controllers with all the control gains $d_{q_{i}}>0$ to be designed.

The objective of control here is to find some appropriate controllers (2.6) such that the solutions of the controlled network (2.5) globally synchronize with the solution of (2.4), in the sense that

$$
\lim _{t \rightarrow \infty}\left\|x_{i}(t)-s(t)\right\|=0, \quad i=1,2, \ldots, N
$$

for any initial conditions. When the controlled complex network (2.5) achieves synchronization, the coupling terms and control inputs will automatically vanish due to the diffusive condition $\sum_{j=1}^{N} G_{i j}=0, i=1,2, \ldots, N$, which indicates that any solution $x_{i}(t)$ of a single vertex is also a solution of the synchronized coupled network.

Throughout the rest of the paper, the following assumption is needed.

Assumption 1. There exists a constant matrix $K$ such that

$$
(x-y)^{T}(f(x, t)-f(y, t)) \leq(x-y)^{T} K \Gamma(x-y) \quad \forall x, y \in \mathbb{R}^{n} .
$$

Note that Assumption 1 is very mild and that many systems, for example, Lorenz system [7], Chen system [54], Lü system [62], recurrent neural networks [49], Chua's 
circuit [7], linear systems, and piecewise linear systems, satisfy this condition if $\partial f_{i} / \partial x_{j}(i, j=1,2, \ldots, n)$ are uniformly bounded and $\Gamma$ is positive definite. Generally speaking, any function in the system satisfying the Lipschitz condition can guarantee Assumption 1. In order to derive the main results, some basic notions and properties from graph theory are introduced.

Let $\mathcal{G}=(\mathcal{V}, \mathcal{E}, G)$ be a weighted directed network of order $N$, with the set of vertices $\mathcal{V}=\left\{v_{1}, v_{2}, \ldots, v_{N}\right\}$, the set of directed edges $\mathcal{E} \subseteq \mathcal{V} \times \mathcal{V}$, and a weighted adjacency matrix $G=\left(G_{i j}\right)_{N \times N}$. A directed edge $\mathcal{E}_{i j}$ in network $\mathcal{G}$ is denoted by the ordered pair of vertices $\left(v_{i}, v_{j}\right)$, where $v_{i}$ and $v_{j}$ are called the terminal and initial vertices, respectively.

Definition 2.1 (see [14]). A network $\mathcal{G}$ is undirected if there is a connection between vertices $i$ and $j$ in $\mathcal{G}$; then $G_{i j}=G_{j i}>0$; otherwise, $G_{i j}=G_{j i}=0$ $(i \neq j, i, j=1,2, \ldots, N)$. A network $\mathcal{G}$ is directed if there is a connection from vertex $j$ to $i$ in $\mathcal{G}$; then $G_{i j}>0$; otherwise $G_{i j}=0$.

Definition 2.2. The in-degree of vertex $i$ is defined by $k_{i}^{\mathrm{in}}=\sum_{j=1, j \neq i}^{N} G_{i j}$, which is the total weight from all the other vertices to vertex $i$. Similarly, the out-degree of vertex $i$ is defined by $k_{i}^{\text {out }}=\sum_{j=1, j \neq i}^{N} G_{j i}$, which is the total weight from vertex $i$ to all the other vertices.

Note that the pinning synchronization over undirected networks considered [54] is a special case of the directed networks in this paper if $G_{i j}=G_{j i}$ for $i, j=1,2, \ldots, N$.

Definition 2.3 (see [14]). A directed (undirected) path from vertex $v_{j}$ to $v_{i}$ is a sequence of edges $\left(v_{i}, v_{i_{1}}\right),\left(v_{i_{1}}, v_{i_{2}}\right), \ldots,\left(v_{i_{l}}, v_{j}\right)$ in the directed (undirected) network with distinct vertices $v_{i_{k}}, k=1,2, \ldots, l$. A directed (undirected) network $\mathcal{G}$ is strongly connected (connected) if between any pair of distinct vertices $v_{i}$ and $v_{j}$ $(i, j=1,2, \ldots, N)$ in $\mathcal{G}$ there is a directed (undirected) path from $v_{i}$ to $v_{j}$. A directed network $\mathcal{G}$ is weakly connected if the direction of the network is ignored and the connectivity is defined as if the network is undirected.

Definition 2.4 (see [14]). The weighted adjacency matrix $G$ in a directed (undirected) network $\mathcal{G}$ is reducible if there is a permutation matrix $P \in \mathbb{R}^{N \times N}$ and an integer $m$ with $1 \leq m \leq N-1$, such that

$$
P^{T} G P=\left(\begin{array}{cc}
\widetilde{G}_{11} & 0 \\
\widetilde{G}_{21} & \widetilde{G}_{22}
\end{array}\right)
$$

where $\widetilde{G}_{11} \in \mathbb{R}^{m \times m}, \widetilde{G}_{21} \in \mathbb{R}^{(N-m) \times m}$, and $\widetilde{G}_{22} \in \mathbb{R}^{(N-m) \times(N-m)}$. If $G$ is not reducible, then $G$ is called irreducible.

Obviously, a nonzero matrix $G$ of order 1 is irreducible. Next, a lemma is given to show the relation between an irreducible matrix and the corresponding strong connectivity in the network.

Lemma 2.5 (Theorem 3.2.1 of [5]; Theorem 6.2.24 of [14]). A matrix $G$ is irreducible if and only if its corresponding network $\mathcal{G}$ is strongly connected.

Lemma 2.5 is very easy to understand. For a strongly connected network, $G$ can not be permutated in the form of the matrix detailed in Definition 2.4, where the 0 matrix denotes that there are no connections from one component to another in the network. Therefore, $G$ is irreducible if the network is strongly connected. Note that strongly connected networks are often used in this paper, and Definition 2.4 provides a theoretical way to justify the strongly connected networks.

Copyright $@$ by SIAM. Unauthorized reproduction of this article is prohibited. 
Definition 2.6 (see [5]). An undirected network is called a tree if it is connected and has no cycles. A directed network is called a directed tree if the underlying network (the direction of the network is ignored as an undirected network) is a tree. A directed tree with a root is a directed network with a vertex $r$ having the property that for each vertex $v$ different from $r$, there is a unique directed path from $r$ to $v$. A directed spanning tree of a network $\mathcal{G}$ is a directed tree, containing all the vertices in $\mathcal{G}$, with a root. A directed forest is a directed network consisting of more directed trees, no two of which have a vertex in common.

Let $\mathcal{S}, \mathcal{P}, \mathcal{W}$, and $\mathcal{F}$ denote the sets of all the strongly connected networks, networks with a directed spanning tree, weakly connected networks, and directed forests, respectively. Then, one has $\mathcal{S} \subseteq \mathcal{P} \subseteq \mathcal{W} \subseteq \mathcal{F}$. For undirected networks, $\mathcal{S}=\mathcal{P}=\mathcal{W}$. So, clearly the study of pinning synchronization on undirected networks is much easier than that on directed networks.

Let $\rho(A)$ be the spectral radius of matrix $A, \lambda_{\max }(F)$ be the largest eigenvalue of matrix $F, F^{T}$ be the transpose of matrix $F, I_{N}$ be the $N$-dimensional identity matrix, and $1_{N} \in \mathbb{R}^{N}$ be a vector with each entry being 1 . For matrices $\widetilde{A}$ and $\widetilde{B}$ with the same order, $\widetilde{A}>\widetilde{B}$ denotes that $\widetilde{A}-\widetilde{B}$ is a positive definite matrix. A matrix $G \in \mathbb{R}^{N \times N}$ is nonnegative if every entry $G_{i j} \geq 0(1 \leq i, j \leq N)$, and a vector $x \in \mathbb{R}^{N}$ is positive if every entry $x_{i}>0(1 \leq i \leq N)$.

Matrix $G$ in this paper is a singular and irreducible Metzler matrix [31]. In this paper, we focus on algebraic graph theory based on network topology and try to understand the basic principles for synchronization in directed complex networks.

Lemma 2.7 (see [7]). If $G$ is irreducible, $G_{i j}=G_{j i} \geq 0$ for $i \neq j$, and $\sum_{j=1}^{N} G_{i j}=$ 0 for all $i=1,2, \ldots, N$, then all eigenvalues of the matrix

$$
\left(\begin{array}{cccc}
G_{11}-\varepsilon & G_{12} & \ldots & G_{1 N} \\
G_{21} & G_{22} & \ldots & G_{2 N} \\
\vdots & \vdots & \ddots & \vdots \\
G_{N 1} & G_{N 2} & \ldots & G_{N N}
\end{array}\right)
$$

are negative for any positive constant $\varepsilon$.

Lemma 2.8 (Theorem 8.4.4 of [14]). Suppose that $A$ is irreducible and nonnegative. Then, $\rho(A)$ is an eigenvalue of $A$ and there is a positive vector $x$ such that $A x=\rho(A) x$.

Lemma 2.9 (Lemma 8.1.21 of [14]). Suppose that $A$ is nonnegative. If the row sums of $A$ are constant, then $\rho(A)=\|A\|_{\infty}$.

LEMma 2.10. For a strongly connected directed network $G$ in the form of (2.2), where $\sum_{j=1}^{N} G_{i j}=0$ with $G_{i j} \geq 0(i \neq j)$, the eigenvalues of $G$ satisfy $0=\lambda_{1}(G)>$ $\mathcal{R}\left(\lambda_{2}(G)\right) \geq \cdots \geq \mathcal{R}\left(\lambda_{N}(G)\right)$, where $\lambda_{i}(G)$ is the ith eigenvalue of $G$ and $\mathcal{R}\left(\lambda_{i}(G)\right)$ denotes the real part of eigenvalue $\lambda_{i}(G), i=1,2, \ldots, N$.

Proof. From the Gersgorin theorem, all the eigenvalues of $G$ are located in the union of $N$ discs given by

$$
\bigcup_{i=1}^{N}\left\{\lambda \in \mathbb{C}:\left\|\lambda-G_{i i}\right\|_{C} \leq \sum_{j=1, j \neq i}^{N} G_{i j}\right\},
$$

where $\mathbb{C}$ is the complex plane and $\|\cdot\|_{C}$ is the Euclidean norm of complex numbers. Since $\sum_{j=1}^{N} G_{i j}=0$, it is easy to see that $\mathcal{R}(\lambda) \leq 0$ for all $i=1,2, \ldots, N$. For 
a strongly connected directed network, $G$ has one eigenvalue 0 with multiplicity 1 [22].

For an undirected or a balanced network, it is well known that $1_{N}$ is not only the left eigenvector but also the right eigenvector of $G$ associated with eigenvalue $0[29,30]$, that is, $G 1_{N}=0_{N}$ and $1_{N}^{T} G=0_{N}^{T}$. However, for a general directed network, $1_{N}$ is not the left eigenvector of $G$ associated with eigenvalue 0 . Since the left eigenvector is very important in determining the final asymptotic states [27], the following property is derived.

LEMMA 2.11. Suppose that $G$ is irreducible and satisfies $\sum_{j=1}^{N} G_{i j}=0$ with $G_{i j} \geq 0(i \neq j)$. Then, there is a positive vector $x$ such that $G^{T} x=0$.

Proof. Choose a positive integer $l$ such that $l+\lambda_{N}(G)>0$ and $l+G_{i i}>0$ for all $i=1,2, \ldots, N$. Then, the matrix $G+l I_{N}$ is nonnegative, and by Lemma $2.9, \rho\left(G+l I_{N}\right)=l$. The matrix $\left(G+l I_{N}\right)^{T}=G^{T}+l I_{N}$ is also nonnegative and $\rho\left(G^{T}+l I_{N}\right)=l$. By Lemma 2.8 , there is a positive vector $x$ such that $\left(G^{T}+l I_{N}\right) x=$ $l x$, and thus $G^{T} x=0$.

LEMma 2.12. Suppose that $G$ is irreducible and satisfies $\sum_{j=1}^{N} G_{i j}=0$ with $G_{i j} \geq$ $0(i \neq j)$. Then there exists a positive definite diagonal matrix $\Xi=\operatorname{diag}\left(\xi_{1}, \xi_{2}, \ldots, \xi_{N}\right)$ such that $\widehat{G}=\frac{1}{2}\left(\Xi G+G^{T} \Xi\right)$ is symmetric and $\sum_{j=1}^{N} \widehat{G}_{i j}=\sum_{j=1}^{N} \widehat{G}_{j i}=0$ for all $i=1,2, \ldots, N$.

Proof. It is clear that $\widehat{G}$ is symmetric, namely, $\widehat{G}_{i j}=\widehat{G}_{j i}$ for all $i, j=1,2, \ldots, N$. From Lemma 2.11, there is a positive vector $\xi=\left(\xi_{1}, \xi_{2}, \ldots, \xi_{N}\right)^{T}$ such that $G^{T} \xi=0$. One has $\xi=\Xi 1_{N}$ and thus $G^{T} \Xi 1_{N}=0$. Therefore, $G^{T} \Xi$ is a matrix, with the sum of the entries in each row being zero. Since $\sum_{j=1}^{N} G_{i j}=0$, one has $\Xi G 1_{N}=0$, and the sum of the entries in each row of $\Xi G$ is also zero. Consequently, the sum of the entries in each row of matrix $\widehat{G}$ is zero. In addition, since $\widehat{G}$ is symmetric, the sum of the entries in each column of matrix $\widehat{G}$ is also zero.

Lemma 2.13 (Schur complement [4]). The linear matrix inequality (LMI)

$$
\left(\begin{array}{cc}
\mathcal{Q}(x) & \mathcal{S}(x) \\
\mathcal{S}(x)^{T} & \mathcal{R}(x)
\end{array}\right)>0
$$

where $\mathcal{Q}(x)=\mathcal{Q}(x)^{T}, \mathcal{R}(x)=\mathcal{R}(x)^{T}$ is equivalent to one of the following conditions:

(i) $\mathcal{Q}(x)>0, \mathcal{R}(x)-\mathcal{S}(x)^{T} \mathcal{Q}(x)^{-1} \mathcal{S}(x)>0$;

(ii) $\mathcal{R}(x)>0, \mathcal{Q}(x)-\mathcal{S}(x) \mathcal{R}(x)^{-1} \mathcal{S}(x)^{T}>0$.

In algebraic graph theory, the lemmas given above are very helpful for deriving synchronization conditions via pinning control on networks, as shown in the following section.

3. Synchronization criteria via pinning control on strongly connected networks. In this section, some general pinning synchronization criteria on strongly connected complex networks are derived. Without loss of generality, we rearrange the order of the vertices in the network so that the first $l$ vertices are controlled.

Then, subtracting (2.4) from (2.5) yields the following error dynamical network:

$$
\begin{aligned}
& \dot{e}_{i}(t)=f\left(x_{i}(t), t\right)-f(s(t), t)+c \sum_{j=1}^{N} G_{i j} \Gamma e_{j}(t)-c d_{i} \Gamma e_{i}(t), \quad i=1,2, \ldots, l \\
& \dot{e}_{i}(t)=f\left(x_{i}(t), t\right)-f(s(t), t)+c \sum_{j=1}^{N} G_{i j} \Gamma e_{j}(t), \quad i=l+1,2, \ldots, N
\end{aligned}
$$

where $e_{i}(t)=x_{i}(t)-s(t), i=1,2, \ldots, N$. 
TheOREm 3.1. Suppose that Assumption 1 holds. The controlled strongly connected network (2.5) is globally synchronized if the following condition is satisfied:

$$
\Xi \otimes\left(K \Gamma+\Gamma^{T} K^{T}\right) / 2+c \widehat{G} \otimes\left(\Gamma+\Gamma^{T}\right) / 2-c(\Xi D) \otimes\left(\Gamma+\Gamma^{T}\right) / 2<0,
$$

where $\otimes$ is the Kronecker product,

$$
D=\operatorname{diag}(\underbrace{d_{1}, \ldots, d_{l}}_{l}, \underbrace{0, \ldots, 0}_{N-l})
$$

$\Xi$ is a positive definite diagonal matrix obtained by Lemma 2.12 , and $\widehat{G}=\frac{1}{2}(\Xi G+$ $\left.G^{T} \Xi\right)$.

Proof. Consider the Lyapunov functional candidate

$$
V(t)=\frac{1}{2} \sum_{i=1}^{N} e_{i}^{T}(t) \xi_{i} e_{i}(t)
$$

where $\Xi=\operatorname{diag}\left(\xi_{1}, \xi_{2}, \ldots, \xi_{N}\right)$ is a positive definite matrix obtained by Lemma 2.12 .

Taking the derivative of $V(t)$ along the trajectories of (3.1) gives

$$
\begin{aligned}
\dot{V} & =\sum_{i=1}^{N} e_{i}^{T}(t) \xi_{i} \dot{e}_{i}(t) \\
& =\sum_{i=1}^{N} e_{i}^{T}(t) \xi_{i}\left[f\left(x_{i}(t), t\right)-f(s(t), t)+c \sum_{j=1}^{N} G_{i j} \Gamma e_{j}(t)\right]-c \sum_{i=1}^{l} d_{i} \xi_{i} e_{i}^{T}(t) \Gamma e_{i}(t) \\
& \leq \sum_{i=1}^{N} e_{i}^{T}(t) \xi_{i}\left[K \Gamma e_{i}(t)+c \sum_{j=1}^{N} G_{i j} \Gamma e_{j}(t)\right]-c \sum_{i=1}^{l} d_{i} \xi_{i} e_{i}^{T}(t) \Gamma e_{i}(t) \\
& =e^{T}(t)[\Xi \otimes(K \Gamma)+c(\widehat{G}-\Xi D) \otimes \Gamma] e(t),
\end{aligned}
$$

where $e(t)=\left(e_{1}^{T}(t), e_{2}^{T}(t), \ldots, e_{N}^{T}(t)\right)^{T}$.

From (3.4), if condition (3.2) is satisfied, the directed network (2.5) is globally synchronized under the given linear feedback pinning controllers.

Assume that $K$ and $\Gamma$ are commutable, and let

$$
\theta=\lambda_{\max }\left(\frac{K+K^{T}}{2}\right)
$$

Note that the above assumption is very reasonable. $\Gamma$ is the inner coupling matrix in (2.1), and $K$ is defined in Assumption 1 to represent the Lipschitz condition. For a positive definite matrix $\Gamma$, one can choose a large $\theta$ such that

$$
(x-y)^{T}(f(x, t)-f(y, t)) \leq \theta(x-y)^{T} \Gamma(x-y) \quad \forall x, y \in \mathbb{R}^{n} .
$$

Here, only $\theta \geq 0$ is considered for simplicity; otherwise, the network is synchronized even without pinning control [54]. The above assumptions are used throughout the paper. 
Corollary 3.2. Suppose that Assumption 1 holds and $\Gamma$ is a positive definite matrix. The controlled strongly connected network (2.5) is globally synchronized if the following condition is satisfied:

$$
C \equiv \theta \Xi+c(\widehat{G}-\Xi D)<0,
$$

where $\Xi$ is a positive definite diagonal matrix obtained by Lemma 2.12, and $\widehat{G}=$ $\frac{1}{2}\left(\Xi G+G^{T} \Xi\right)$.

Proof. From (3.5) and the fact that $\Gamma$ is positive definite, it can be easily verified that

$$
\begin{aligned}
\Xi \otimes & \left(K \Gamma+\Gamma^{T} K^{T}\right) / 2+c \widehat{G} \otimes\left(\Gamma+\Gamma^{T}\right) / 2-c(\Xi D) \otimes\left(\Gamma+\Gamma^{T}\right) / 2 \\
& \leq \Xi \otimes\left(K+K^{T}\right) / 2 \Gamma+c \widehat{G} \otimes \Gamma-c(\Xi D) \otimes \Gamma \\
& \leq(\theta \Xi+c \widehat{G}-c \Xi D) \otimes \Gamma .
\end{aligned}
$$

If $\theta \Xi+c(\widehat{G}-\Xi D)<0$ and $\Gamma>0$, then it follows that condition (3.2) is satisfied [20].

Let

$$
C=\theta \Xi+c(\widehat{G}-\Xi D)=\left(\begin{array}{cc}
C_{11}-\widetilde{D} & C_{12} \\
C_{12}^{T} & \widetilde{C}
\end{array}\right),
$$

where $C_{11}$ and $C_{12}$ are matrices with appropriate dimensions, $\widetilde{D}=\operatorname{diag}\left(\xi_{1} d_{1}, \ldots, \xi_{l} d_{l}\right)$, and $\widetilde{C}$ is obtained by removing the $1,2, \ldots, l$ row-column pairs of matrix $C$ or $\theta \Xi+c \widehat{G}$.

Corollary 3.3. Suppose that Assumption 1 holds and $\Gamma$ is a positive definite matrix. The controlled strongly connected network (2.5) is globally synchronized if one of the following conditions are satisfied:

$$
c>\frac{\lambda_{\max }(\theta \Xi)}{\left|\lambda_{\max }(\widehat{G}-\Xi D)\right|}
$$

where $\Xi$ is a positive definite diagonal matrix obtained by Lemma 2.12, and $\widehat{G}=$ $\frac{1}{2}\left(\Xi G+G^{T} \Xi\right)$

(ii)

$$
\widetilde{C}<0,
$$

where the control gain matrix is chosen by $\widetilde{D}>\lambda_{\max }\left(C_{11}-C_{12} \widetilde{C}^{-1} C_{12}^{T}\right) I_{l}$.

Proof. By Lemma 2.12, one knows that $\widehat{G}_{i j}=\widehat{G}_{j i}$ for $i \neq j$ and $\sum_{j=1}^{N} \widehat{G}_{i j}=0$. Since $\xi$ is positive, by Lemma 2.7 , it is easy to see that $\widehat{G}-\Xi D^{*}$ is negative definite, where $D^{*}$ corresponds to having only one controller, i.e., $l=1$. Then one obtains $\lambda_{\max }(\widehat{G}-\Xi D) \leq \lambda_{\max }\left(\widehat{G}-\Xi D^{*}\right)<0$. From (3.8), one has $\theta \Xi+c(\widehat{G}-\Xi D) \leq$ $\lambda_{\max }(\theta \Xi)+c \lambda_{\max }(\widehat{G}-\Xi D)<0$, so (3.6) is satisfied. The proof of (i) is completed. Condition (ii) can be proved by using Lemma 2.13, as in [54].

Normally, the theoretical coupling strength and control gain matrix given in (3.8) and (3.9) are very conservative - usually much larger than the needed values. The adaptive technique $[7,47,57,61]$ can be used here to choose smaller coupling strength and control gain. Detailed results can be found in [54] and thus are omitted here.

Copyright (c) by SIAM. Unauthorized reproduction of this article is prohibited. 
Remark 1. In Corollary 3.3, condition (i) provides a way to choose the coupling strength when the network structure and the pinning scheme are given; condition (ii) shows that synchronization can be reached in a lower-dimensional setting by appropriately choosing the control gain matrix when the network structure and the coupling strength are fixed. If the coupling strength is very large and satisfies condition (3.8), then by controlling very few vertices, the network can achieve synchronization. In addition, note that $\widetilde{C}$ is obtained by removing the $1,2, \ldots, l$ row-column pairs of matrix $\theta \Xi+c \widehat{G}$, which is independent of matrix $D$. Therefore, the pinning controllers are not involved in (3.9), which provides some guidance for choosing appropriate pinning schemes. After choosing the positive definite matrix $\widetilde{C}$, one can design the control gain matrix such that $\widetilde{D}>\lambda_{\max }\left(C_{11}-C_{12} \widetilde{C}^{-1} C_{12}^{T}\right) I_{l}$. From (3.9), it is easy to see that the network more likely can reach synchronization if the vertices with large out-degrees are pinned, which means that there are many nonzero entries in $C_{12}^{T}$.

Let $C=\left(C_{i j}\right)_{N \times N}$ in (3.6). Then, one has

$$
C_{i j}=c \frac{\xi_{i} G_{i j}+\xi_{j} G_{j i}}{2}(i \neq j),
$$

$C_{i i}=\theta \xi_{i}-c \xi_{i} k_{i}^{\text {in }}-c \xi_{i} d_{i}(1 \leq i \leq l)$, and $C_{i i}=\theta \xi_{i}-c \xi_{i} k_{i}^{\text {in }}(l+1 \leq i \leq N)$.

CoROllary 3.4. Suppose that Assumption 1 holds and $\Gamma$ is a positive definite matrix. To satisfy condition (3.6), it is necessary that

$$
\begin{aligned}
d_{i} & >\frac{\theta}{c}-k_{i}^{\text {in }}, \quad 1 \leq i \leq l, \\
k_{i}^{\text {in }} & >\frac{\theta}{c}, \quad l+1 \leq i \leq N .
\end{aligned}
$$

Proof. To ensure $C<0$ in (3.6), it is necessary that $C_{i i}<0$.

Remark 2. In Corollary 3.4, the in-degree of vertex $i$ without control should be greater than a critical value, that is, $k_{i}^{\text {in }}>\frac{\theta}{c}$. Therefore, it is easy to see that under condition (3.6), the vertices with very small in-degrees less than $\theta / c$ should be controlled first, where condition (3.10) is not satisfied. Note that some vertices with very small in-degrees receive very little information from all the other vertices, and therefore it is inadequate to drive these small in-degree vertices in order to achieve network synchronization. Condition (3.6) in Corollary 3.2 is very simple; however, how to choose the minimal number of pinning controllers under a fixed network structure with a fixed coupling strength is still a challenging research problem.

4. Synchronization criteria via pinning control on networks with a directed spanning tree. In the previous section, pinning synchronization on strongly connected networks was studied. Considering the reference vertex with state $s(t)$ in (2.4) as a virtual leader in the network, now we will change our focus to pinning synchronization on each strongly connected component of a general network, where the network contains a directed spanning tree with the virtual leader as the root.

The dynamics of (2.4) and (2.5) can be rewritten in compact form as follows:

$$
\dot{y}_{i}(t)=f\left(y_{i}(t), t\right)+c \sum_{j=1}^{N} \widetilde{G}_{i j} \Gamma y_{j}(t), \quad i=1, \quad l+1,2, \ldots, N+1,
$$

where $y_{1}=s, y_{i+1}=x_{i}$ for $i=1,2, \ldots, N$,

$$
\widetilde{G}=\left(\begin{array}{cc}
0 & O \\
d & G-D
\end{array}\right),
$$


$O$ is a 0 matrix with appropriate dimensions, and

$$
d=(\underbrace{d_{1}, \ldots, d_{l}}_{l}, \underbrace{0, \ldots, 0}_{N-l})^{T} .
$$

In the following, suppose that the controllers are added to the $q_{1}, q_{2}, \ldots, q_{l}$ vertices, and so

$$
\widetilde{G}=\left(\begin{array}{cc}
0 & O \\
\widetilde{d} & G-\widetilde{D}
\end{array}\right)
$$

where

$$
\widetilde{d}=(\ldots, \underbrace{d_{1}}_{q_{1}}, 0, \ldots, 0, \underbrace{d_{2}}_{q_{2}}, 0, \ldots, 0, \underbrace{d_{l}}_{q_{l}}, \ldots)^{T}
$$

and $\widetilde{D}$ is a diagonal matrix with diagonal elements $\widetilde{D}_{i i}=\widetilde{d}_{i}, i=1,2, \ldots, N$.

Since the matrix $G$ is irreducible, as discussed in the previous section, the network structure $\widetilde{G}$ has a directed spanning tree as long as at least one vertex is controlled by the leader $s$, which is a root in the directed spanning tree [34]. In order to derive the main result, the following Frobenius normal form is needed.

Lemma 4.1 (see [5]). Let $\widetilde{G}$ be a matrix of order $N+1$. Then, there exists a permutation matrix $P$ of order $N+1$ and an integer $m \geq 1$, such that

$$
P^{T} \widetilde{G} P=\left(\begin{array}{cccc}
\widetilde{G}_{1} & O & \cdots & O \\
\widetilde{G}_{21} & \widetilde{G}_{2} & \cdots & O \\
\vdots & \vdots & \ddots & O \\
\widetilde{G}_{m 1} & \widetilde{G}_{m 2} & \cdots & \widetilde{G}_{m}
\end{array}\right),
$$

where $\widetilde{G}_{1} \in \mathbb{R}^{p_{1} \times p_{1}}, \widetilde{G}_{2} \in \mathbb{R}^{p_{2} \times p_{2}}, \ldots, \widetilde{G}_{m} \in \mathbb{R}^{p_{m} \times p_{m}}$ are square irreducible matrices which are uniquely determined to within simultaneous permutation of their lines, but their ordering is not necessarily unique.

Definition 4.2 (see [5]). Let $\widetilde{\mathcal{G}}$ be a directed network and $\widetilde{\mathcal{G}}_{1}, \widetilde{\mathcal{G}}_{2}, \ldots, \widetilde{\mathcal{G}}_{m}$ be the strongly connected components of $\widetilde{\mathcal{G}}$ with connection matrices $\widetilde{G}_{1}, \widetilde{G}_{2}, \ldots, \widetilde{G}_{m}$. Then $\widetilde{\mathcal{G}}^{*}$ is a condensation network of $\widetilde{\mathcal{G}}$ if there is a connection from a vertex in $\mathcal{V}\left(\widetilde{\mathcal{G}}_{j}\right)$ to a vertex in $\mathcal{V}\left(\widetilde{\mathcal{G}}_{i}\right)(i \neq j)$, and then the weight $\widetilde{G}_{i j}^{*}>0$; otherwise, $\widetilde{G}_{i j}^{*}=0$ for $i, j=1,2, \ldots, m$, where $\widetilde{G}_{i i}^{*}=0$ for $i=1,2, \ldots, m$.

Note that the condensation network $\widetilde{\mathcal{G}}^{*}$ of any directed network $\widetilde{\mathcal{G}}$ has no closed directed walks [5], where a directed (undirected) walk from vertex $v_{j}$ to $v_{i}$ is a sequence of edges $\left(v_{i}, v_{i_{1}}\right),\left(v_{i_{1}}, v_{i_{2}}\right), \ldots,\left(v_{i_{l}}, v_{j}\right)$ in the directed (undirected) network with vertices $v_{i_{k}}, k=1,2, \ldots, l$.

Lemma 4.3 (see [41]). For every $i=2,3, \ldots, m$, there is an integer $j<i$ such that $\widetilde{G}_{i j}^{*}>0$ if and only if the directed network $\widetilde{\mathcal{G}}$ contains a directed spanning tree.

One can change the ordering of the vertices to obtain the Frobenius normal form (4.1). Without loss of generality, assume $\mathcal{G}$ is in the Frobenius normal form. Then $\widetilde{G}_{1}=0$ because the virtual reference agent is not influenced by any agent of the network. Let $\widetilde{G}_{j}=A_{j}+B_{j}$, where $A_{j}$ is a zero row-sums matrix and $B_{j} \leq 0$ is a diagonal matrix. By Lemma 2.11, there exists a positive vector $\Pi_{j}=\left(\Pi_{j 1}, \Pi_{j 2}, \ldots, \Pi_{j p_{j}}\right)$ with appropriate dimensions such that $\Pi_{j}^{T} A_{j}=0$. Let $e=\left(\widetilde{e}_{2}^{T}, \widetilde{e}_{3}^{T}, \ldots, \widetilde{e}_{m-1}^{T}\right)^{T}$, where $\widetilde{e}_{j} \in R^{p_{j} \times 1}$ for $j=2, \ldots, m$. 
LEMMA 4.4. Let

$$
\bar{G}=\left(\begin{array}{cccc}
\widetilde{G}_{2} & O & \cdots & O \\
\widetilde{G}_{32} & \widetilde{G}_{3} & \cdots & O \\
\vdots & \vdots & \ddots & O \\
\widetilde{G}_{m 2} & \widetilde{G}_{m 3} & \cdots & \widetilde{G}_{m}
\end{array}\right)
$$

If there exist positive definite diagonal matrices $\widetilde{\Pi}_{j}=\operatorname{diag}\left(\Pi_{j 1}, \Pi_{j 2}, \ldots, \Pi_{j p_{j}}\right)$ for $j=2, \ldots, m$, such that

$$
\widetilde{\Pi}_{j} \widetilde{G}_{j}+\widetilde{G}_{j}^{T} \widetilde{\Pi}_{j}<0
$$

then there exists a positive definite diagonal matrix $\Delta=\operatorname{diag}\left(\Delta_{2} I_{p_{2}}, \ldots, \Delta_{m} I_{p_{m}}\right)$, such that

$$
\Delta \widetilde{\Pi} \bar{G}+\bar{G}^{T} \widetilde{\Pi} \Delta<0
$$

where $\widetilde{\Pi}=\operatorname{diag}\left(\widetilde{\Pi}_{2}, \ldots, \widetilde{\Pi}_{m}\right)$ and $\Delta_{j}$ are positive constants for $j=2, \ldots, m$.

Proof. Let $Q_{i}$ be of the form

$$
Q_{i}=\left(\begin{array}{cccc}
\Delta_{2}\left(\widetilde{\Pi}_{2} \widetilde{G}_{2}+\widetilde{G}_{2}^{T} \widetilde{\Pi}_{2}\right) & \Delta_{3} \widetilde{\Pi}_{3} \widetilde{G}_{32} & \cdots & \Delta_{i} \widetilde{\Pi}_{i} \widetilde{G}_{i 2} \\
\Delta_{3} \widetilde{\Pi}_{3} \widetilde{G}_{32} & \Delta_{3}\left(\widetilde{\Pi}_{3} \widetilde{G}_{3}+\widetilde{G}_{3}^{T} \widetilde{\Pi}_{3}\right) & \cdots & \Delta_{i} \widetilde{\Pi}_{i} \widetilde{G}_{i 3} \\
\vdots & \vdots & \ddots & \vdots \\
\Delta_{i} \widetilde{\Pi}_{i} \widetilde{G}_{i 2} & \Delta_{i} \widetilde{\Pi}_{i} \widetilde{G}_{i 3} & \cdots & \Delta_{i}\left(\widetilde{\Pi}_{i} \widetilde{G}_{i}+\widetilde{G}_{i}^{T} \widetilde{\Pi}_{i}\right)
\end{array}\right) .
$$

Then, $Q_{m}=\Delta \widetilde{\Pi} \bar{G}+\bar{G}^{T} \widetilde{\Pi} \Delta$. From (4.4), it is easy to see that $Q_{2}<0$. Next, the lemma is proved by induction. Suppose that $Q_{i}<0$. We need to show that $Q_{i+1}<0$. By Lemma 2.13, $Q_{i+1}<0$ is equivalent to $\widetilde{\Pi}_{i+1} \widetilde{G}_{i+1}+\widetilde{G}_{i+1}^{T} \widetilde{\Pi}_{i+1}<0$, and we have

$$
Q_{i}-\Delta_{i+1} \widetilde{Q}_{i+1}\left(\widetilde{\Pi}_{i+1} \widetilde{G}_{i+1}+\widetilde{G}_{i+1}^{T} \widetilde{\Pi}_{i+1}\right)^{-1} \widetilde{Q}_{i+1}^{T}<0,
$$

where $\widetilde{Q}_{i+1}^{T}=\widetilde{\Pi}_{i+1}\left(\widetilde{G}_{(i+1) 2}, \widetilde{G}_{(i+1) 3}, \ldots, \widetilde{G}_{(i+1) i}\right)$. If $\Delta_{i+1}$ is sufficiently smaller than $\Delta_{j}$ for $j<i+1$, then (4.7) can be satisfied. Therefore, by choosing $\Delta_{i+1}$ to be sufficiently smaller than $\Delta_{j}$ for $j<i+1$, condition (4.7) can be satisfied.

TheOrem 4.5. Suppose that Assumption 1 holds and $\Gamma$ is a positive definite matrix. The controlled network with a directed spanning tree (2.5) is globally synchronized if the following condition is satisfied:

$$
2 \theta \widetilde{\Pi}_{j}+c\left(\widetilde{\Pi}_{j} \widetilde{G}_{j}+\widetilde{G}_{j}^{T} \widetilde{\Pi}_{j}\right)<0, \quad j=2, \ldots, m .
$$

Proof. Consider the Lyapunov functional candidate

$$
V(t)=\sum_{i=2}^{m} \Delta_{i} \widetilde{e}_{i}^{T}(t) \widetilde{\Pi}_{i} \widetilde{e}_{i}(t)
$$

where $\widetilde{\Pi}_{i}=\operatorname{diag}\left(\Pi_{i 1}, \Pi_{i 2}, \ldots, \Pi_{i p_{i}}\right)$ and $\Delta_{i}$ are positive constants for $j=2, \ldots, m$. 
Taking the derivative of $V(t)$ gives

$$
\begin{aligned}
\dot{V} & =2 \sum_{i=2}^{m} \Delta_{i} \widetilde{e}_{i}^{T}(t) \widetilde{\Pi}_{i} \dot{\tilde{e}}_{i}(t) \\
& \leq 2 \sum_{i=2}^{m} \Delta_{i} \widetilde{e}_{i}^{T}(t) \widetilde{\Pi}_{i}\left[\theta\left(I_{p_{i}} \otimes \Gamma\right) \widetilde{e}_{i}(t)+c \sum_{j=1}^{i-1}\left(\widetilde{G}_{i j} \otimes \Gamma\right) \widetilde{e}_{j}(t)+c\left(\widetilde{G}_{i} \otimes \Gamma\right) \widetilde{e}_{i}(t)\right] \\
& =e^{T}(t)\left[(2 \theta \Delta \widetilde{\Pi}) \otimes \Gamma-c\left(\Delta \widetilde{\Pi} \bar{G}+\bar{G}^{T} \widetilde{\Pi} \Delta\right) \otimes \Gamma\right] e(t) \\
& =e^{T}(t)\left\{\left[\Delta \widetilde{\Pi}\left(c \bar{G}+\theta I_{N}\right)+\left(c \bar{G}++\theta I_{N}\right)^{T} \widetilde{\Pi} \Delta\right] \otimes \Gamma\right\} e(t),
\end{aligned}
$$

where $e(t)=\left(\widetilde{e}_{2}^{T}(t), \widetilde{e}_{3}^{T}(t), \ldots, \widetilde{e}_{m}^{T}(t)\right)^{T}$. By Lemma 4.4 and $(4.8)$, the proof is completed.

Remark 3. The condition (4.8) is very simple, where the study of the whole network synchronization is reduced to the investigation of the strongly connected components in the network.

Let $r_{1}=0, r_{j}=\sum_{k=2}^{j} p_{k}, A_{j}=\left(A_{j k o}\right)_{p_{j} \times p_{j}}$, and $B_{j}=\operatorname{diag}\left(B_{j 1}, \ldots, B_{j p_{j}}\right)$ for $j=2, \ldots, m ; k, o=1, \ldots, p_{j}$. Then, one has $B_{j k}=-\sum_{s=1}^{r_{j-1}} G_{\left(r_{j-1}+k\right) s}-\widetilde{d}_{r_{j-1}+k}$ for $k=1, \ldots, p_{j} ; j=2, \ldots, m$.

Definition 4.6. Let $C_{j k} \equiv \sum_{s=1}^{r_{j-1}} G_{\left(r_{j-1}+k\right) s}$ be the total weights from the other strongly connected components to the kth vertex in the $j$ th component for $j=$ $2, \ldots, m, k=1, \ldots, p_{j}$. Let $A_{j k k} \equiv \sum_{s=r_{j-1}+1}^{r_{j}} G_{\left(r_{j-1}+k\right) s}$ be the total weights from the other vertices in the $j$ th component to the $k$ th vertex in the $j$ th component for $j=2, \ldots, m, k=1, \ldots, p_{j} . C_{j k}$ and $A_{j k k}$ are called the outer in-degree and inner in-degree of the $k$ th vertex in the $j$ th component, respectively. Similarly, $\widehat{C}_{j} \equiv$ $\sum_{k=1}^{p_{j}} \sum_{s=1}^{r_{j-1}} G_{\left(r_{j-1}+k\right) s}$ and $\widehat{A}_{j} \equiv \sum_{k=1}^{p_{j}} \sum_{s=r_{j-1}+1}^{r_{j}} G_{\left(r_{j-1}+k\right) s}$ are called the outer in-degree and inner in-degree of the $j$ th component, respectively.

Let $\bar{A}_{j}=\frac{1}{2}\left(\widetilde{\Pi}_{j} A_{j}+A_{j}^{T} \widetilde{\Pi}_{j}\right), \bar{D}_{j}=\operatorname{diag}\left(\widetilde{d}_{r_{j-1}+1}, \ldots, \widetilde{d}_{r_{j}}\right), C_{j}=\operatorname{diag}\left(C_{j 1}, \ldots, C_{j p_{j}}\right)$ for $j=2,3, \ldots, m$.

Corollary 4.7. Suppose that Assumption 1 holds and $\Gamma$ is a positive definite matrix. The controlled network with a directed spanning tree (2.5) is globally synchronized if the following condition is satisfied:

$$
\theta \widetilde{\Pi}_{j}+c\left(\bar{A}_{j}-\widetilde{\Pi}_{j} C_{j}-\widetilde{\Pi}_{j} \bar{D}_{j}\right)<0, \quad j=2, \ldots, m .
$$

Remark 4. $\bar{A}_{j}$ is a zero row-column-sum symmetric matrix. By Lemma 2.7, $\bar{A}_{j}-\widetilde{\Pi}_{j} C_{j}-\widetilde{\Pi}_{j} \bar{D}_{j}<0$ if and only if $C_{j} \neq 0$ or $\bar{D}_{j} \neq 0$, which means that if there are no connections from all the other strongly connected components and the leader to a particular strongly connected component, then synchronization in this component can not be achieved. This result is easy to understand since the vertices in this component can not receive any information from the leader.

Remark 5. If $\bar{D}_{j}=0$ for some $2 \leq j \leq m$, then in order to reach synchronization, one must have $C_{j} \neq 0$ and $\theta \widetilde{\Pi}_{j}+c\left(\bar{A}_{j}-\widetilde{\Pi}_{j} C_{j}\right)<0$, which implies that if the outer in-degree of a strongly connected component is very high, then synchronization can be easily achieved in this component. If $C_{j}=0$ for some $2 \leq j \leq m$, then the $j$ th strongly connected component must be controlled in order to achieve network synchronization. 
ASSUMPTION 2. Each strongly connected component, to which there are no connections from all the other strongly connected components, is under the control of pinning controllers (2.6).

COROLlary 4.8. Suppose that Assumptions 1 and 2 hold and that $\Gamma$ is a positive definite matrix. The controlled network with a directed spanning tree (2.5) is globally synchronized if one of the following conditions is satisfied:

(i)

$$
c>\max _{2 \leq j \leq m} \frac{\lambda_{\max }\left(\theta \widetilde{\Pi}_{j}\right)}{\left|\lambda_{\max }\left(\bar{A}_{j}-\widetilde{\Pi}_{j} C_{j}-\widetilde{\Pi}_{j} \bar{D}_{j}\right)\right|},
$$

(ii) there exist some controllers with large gains, such that

$$
\Omega_{j}<0
$$

where $\Omega_{j}$ is obtained by removing the row-column pairs of the controlled vertices in matrix $\theta \widetilde{\Pi}_{j}+c\left(\bar{A}_{j}-\widetilde{\Pi}_{j} C_{j}-\widetilde{\Pi}_{j} \bar{D}_{j}\right)$.

The proof can be completed by the same approach as in Corollary 3.3.

Corollary 4.9. Suppose that Assumption 1 holds and $\Gamma$ is a positive definite matrix. The first $i$ strongly connected components of the controlled network with a directed spanning tree (2.5) are globally synchronized if the following condition is satisfied:

$$
2 \theta \widetilde{\Pi}_{j}+c\left(\widetilde{\Pi}_{j} \widetilde{G}_{j}+\widetilde{G}_{j}^{T} \widetilde{\Pi}_{j}\right)<0, j \leq i, i=1,2, \ldots, N .
$$

The derived condition in Corollary 4.9 can be used to study partial synchronization [48] of the whole network.

5. Synchronization criteria via pinning control on general complex networks. This section proposes an algorithm for pinning synchronization on general complex networks, including weakly connected networks and directed forests, as follows.

Pinning scheme algorithm.

(1) Reorder the vertices to obtain the Frobenius normal form (4.3). Initially, let $j=2$ and then go to step (2).

(2) If (4.11) is satisfied when $\bar{D}_{j}=0$, the $j$ th strongly connected component can achieve synchronization without control. If so, go to step (6); otherwise, turn to step (3).

(3) If the outer in-degree of every vertex in the $j$ th strongly connected component is 0 , i.e., $C_{j}=0$, then this $j$ th component must be controlled through the gain matrix $\bar{D}_{j}$. Then, go to step (4); otherwise, turn to step (6).

(4) For the $i$ th vertex in the $j$ th strongly connected component, if its in-degree $k_{r_{j-1}+i}^{\text {in }} \leq \frac{\theta}{c}$, then this vertex must be controlled. Then, go to step (5).

(5) Find the controllers such that (4.13) can be satisfied as discussed in sections 3 and 4 , and then go to step (6).

(6) If $j<m$, then set $j=j+1$ and go to step (2); otherwise, stop.

Remark 6. If (4.11) is satisfied without control, then this strongly connected component can achieve synchronization. Suppose that a strongly connected component does not receive information from all the other strongly connected components. By Lemma 4.3, this component must be controlled so as to form a spanning tree as shown in step (3). Note that the directed network $\bar{G}$ in (4.3) contains a directed 
spanning tree if and only if, for each $j, C_{j} \neq 0$ or $\bar{D}_{j} \neq 0$, which means that there are connections from all the other strongly connected components or the leader to the $j$ th strongly connected component. Steps (4) and (5) are the pinning control schemes on the $j$ th strongly connected component, which was discussed in section 3 .

In this section, an algorithm for studying the pinning synchronization on general complex networks is proposed. Note that a general complex network can be composed of many strongly connected components. If there are no connections from all the other strongly connected components to a particular strongly connected component, then this component must be controlled. After that, it is easy to see that the whole network forms a directed spanning tree with the leader being the root. Then each vertex in the network can receive information from the root and synchronization may be achieved in a successive way.

6. Simulation examples. In order to clearly show the theoretical results in this paper, synchronization via pinning control on a small-size network is first given in this section. Then, to verify the proposed algorithm in this paper, synchronization via pinning control on a large-size scale-free network is performed.

Consider a complex network (2.3) that consists of $N$ identical Chen systems [54], described by

$$
\begin{gathered}
\dot{x}_{i}(t)=f\left(x_{i}(t), t\right)+c \sum_{j=1}^{N} G_{i j} \Gamma x_{j}(t), \\
i=1,2, \ldots, N,
\end{gathered}
$$

where

$$
f\left(x_{i}, t\right)=\left\{\begin{array}{l}
35\left(x_{i 2}-x_{i 1}\right) \\
-7 x_{i 1}-x_{i 1} x_{i 3}+28 x_{i 2} \\
x_{i 1} x_{i 2}-3 x_{i 3}
\end{array}\right.
$$

and $\theta=31$ [54].

6.1. Synchronization via pinning control on a simple toy network. Choose $\Gamma=\operatorname{diag}(1,2,1), c=12$, and $N=10$. The network structure is shown in Figure 6.1. The vertices of the network are ordered from 1 to 10 , with a virtual leader $s$, and the network is composed of 5 strongly connected components as shown in Figure 6.1. From Figure 6.1, one has

$$
\widetilde{G}=\left(\begin{array}{cc}
0 & O \\
\widetilde{d} & \bar{G}
\end{array}\right)
$$

and it is easy to see that the matrix $\bar{G}$ in (4.3) is already in the Frobenius normal form, with $\widetilde{G}_{2}=0$,

$$
\begin{gathered}
\widetilde{G}_{3}=\left(\begin{array}{cccc}
-3 & 0 & 0 & 3 \\
6 & -6 & 0 & 0 \\
6 & 2 & -8 & 0 \\
0 & 0 & 6 & -6
\end{array}\right), \quad \widetilde{G}_{4}=\left(\begin{array}{cc}
-7 & 4 \\
4 & -8
\end{array}\right), \quad \widetilde{G}_{5}=\left(\begin{array}{ccc}
-5 & 0 & 3 \\
2 & -2 & 0 \\
0 & 2 & -5
\end{array}\right), \\
\widetilde{G}_{43}=\left(\begin{array}{llll}
0 & 0 & 3 & 0 \\
0 & 0 & 0 & 4
\end{array}\right), \quad \widetilde{G}_{52}=\left(\begin{array}{l}
0 \\
0 \\
3
\end{array}\right), \quad \text { and } \quad \widetilde{G}_{53}=\left(\begin{array}{llll}
2 & 0 & 0 & 0 \\
0 & 0 & 0 & 0 \\
0 & 0 & 0 & 0
\end{array}\right) .
\end{gathered}
$$




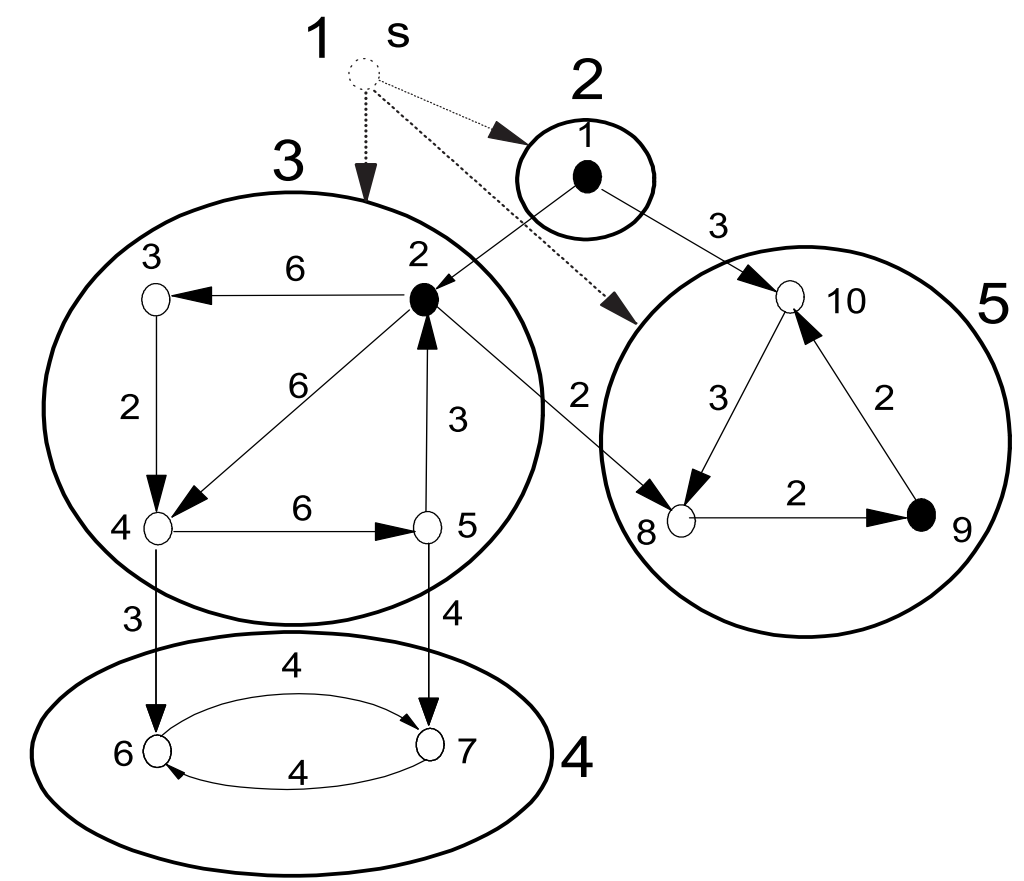

FIG. 6.1. Structure of a small network. The vertices in the big circles are in the same strongly connected components. The dashed and white vertices are the leader and uncontrolled vertices, respectively. The solid vertices are the pinned vertices.

The outer in-degrees of strongly connected components 2 and 3 are zero. So these two components must be controlled, as illustrated in Figure 6.1.

For $j=2$, one has $C_{2}=\bar{A}_{2}=0$. From (4.11), if $d_{1}>\theta / c \approx 2.5833$, then the second strongly connected component can reach synchronization.

For $j=3$, by simple computation, one obtains $C_{3}=0, \widetilde{\Pi}_{3}=\operatorname{diag}(8,1,3,4)$, and

$$
\bar{A}_{3}=\left(\begin{array}{cccc}
-24 & 3 & 9 & 12 \\
3 & -6 & 3 & 0 \\
9 & 3 & -24 & 12 \\
12 & 0 & 12 & -24
\end{array}\right)
$$

Thus, condition (4.11) is

$$
\left(\begin{array}{cccc}
-24+8 \theta / c & 3 & 9 & 12 \\
3 & -6+\theta / c & 3 & 0 \\
9 & 3 & -24+3 \theta / c & 12 \\
12 & 0 & 12 & -24+4 \theta / c
\end{array}\right)-\widetilde{\Pi}_{3} \bar{D}_{3}<0 .
$$

Since

$$
\left(\begin{array}{ccc}
-6+\theta / c & 3 & 0 \\
3 & -24+3 \theta / c & 12 \\
0 & 12 & -24+4 \theta / c
\end{array}\right)<0
$$

Copyright $@$ by SIAM. Unauthorized reproduction of this article is prohibited. 
by (4.13), the third strongly connected component can achieve synchronization if vertex 2 is controlled. By Lemma 2.13, one has $d_{2}>18.2667$.

For $j=4$, one obtains $\widetilde{\Pi}_{4}=I_{2}$ and $C_{4}=\operatorname{diag}(3,4)$. If $\bar{D}_{4}=0$, then $\theta / c+$ $\bar{A}_{4}-C_{4}<0$. Thus, the condition (4.11) is satisfied without control, and the fourth strongly connected component can reach synchronization without control.

For $j=5$, by simple computation, one obtains $C_{5}=\operatorname{diag}(2,0,3), \widetilde{\Pi}_{5}=\operatorname{diag}(2,3,3)$, and

$$
\bar{A}_{5}=\left(\begin{array}{ccc}
-6 & 3 & 3 \\
3 & -6 & 3 \\
3 & 3 & -6
\end{array}\right)
$$

Thus, condition (4.11) is

$$
\left(\begin{array}{ccc}
-10+2 \theta / c & 3 & 3 \\
3 & -6+3 \theta / c & 3 \\
3 & 3 & -15+3 \theta / c
\end{array}\right)-\widetilde{\Pi}_{3} \bar{D}_{3}<0 .
$$

By Corollary 3.4, one has $k_{9}^{\text {in }}=2<\theta / c$; thus $-6+3 \theta / c>0$, which implies that vertex 9 must be controlled. Since

$$
\left(\begin{array}{cc}
-10+2 \theta / c & 3 \\
3 & -15+3 \theta / c
\end{array}\right)<0
$$

if vertex 9 is controlled and one chooses $d_{9}>2.665$, from (4.13) and by Lemma 2.13, (4.11) is satisfied. So synchronization can be reached on the fifth strongly connected component.

Therefore, in the network (6.1), if three vertices, 1, 2, and 9 (solid vertices in Figure 6.1) are controlled with control gains $d_{1}>2.5833, d_{2}>18.2667$, and $d_{9}>$ 2.665 , then the synchronization can be achieved on the whole network, as illustrated in Figure 6.2.

Remark 7. Suppose that a network is composed of some strongly connected components as in (4.2). If the outer in-degrees of all the vertices in some strongly connected components are zero, then these components must be controlled since they do not receive any information from other components, and therefore synchronization can not be reached without control. In the above example, for this reason, the second and third strongly connected components must be controlled. Vertex 2 in the third strongly connected component should be controlled since it transmits a large amount of information to the other vertices. So, by Corollary 3.4, synchronization can be easily achieved if such vertices with large out-degrees are controlled. If a strongly connected component, for example, the fourth strongly component in Figure 6.1, receives a large amount of information from the other components, then it can reach synchronization even without control. Though a strongly connected component, like the fifth strongly component in Figure 6.1, receives a lot of information from the other strongly connected components, it can not achieve synchronization if vertex 9 is not controlled. Vertex 9 receives very little information from the other vertices, and hence it is difficult or inadequate to drive vertex 9 to synchronize with other vertices, and thus it must be controlled. Therefore, in order to reach network synchronization, the vertices with large out-degree or small in-degree should be controlled. 

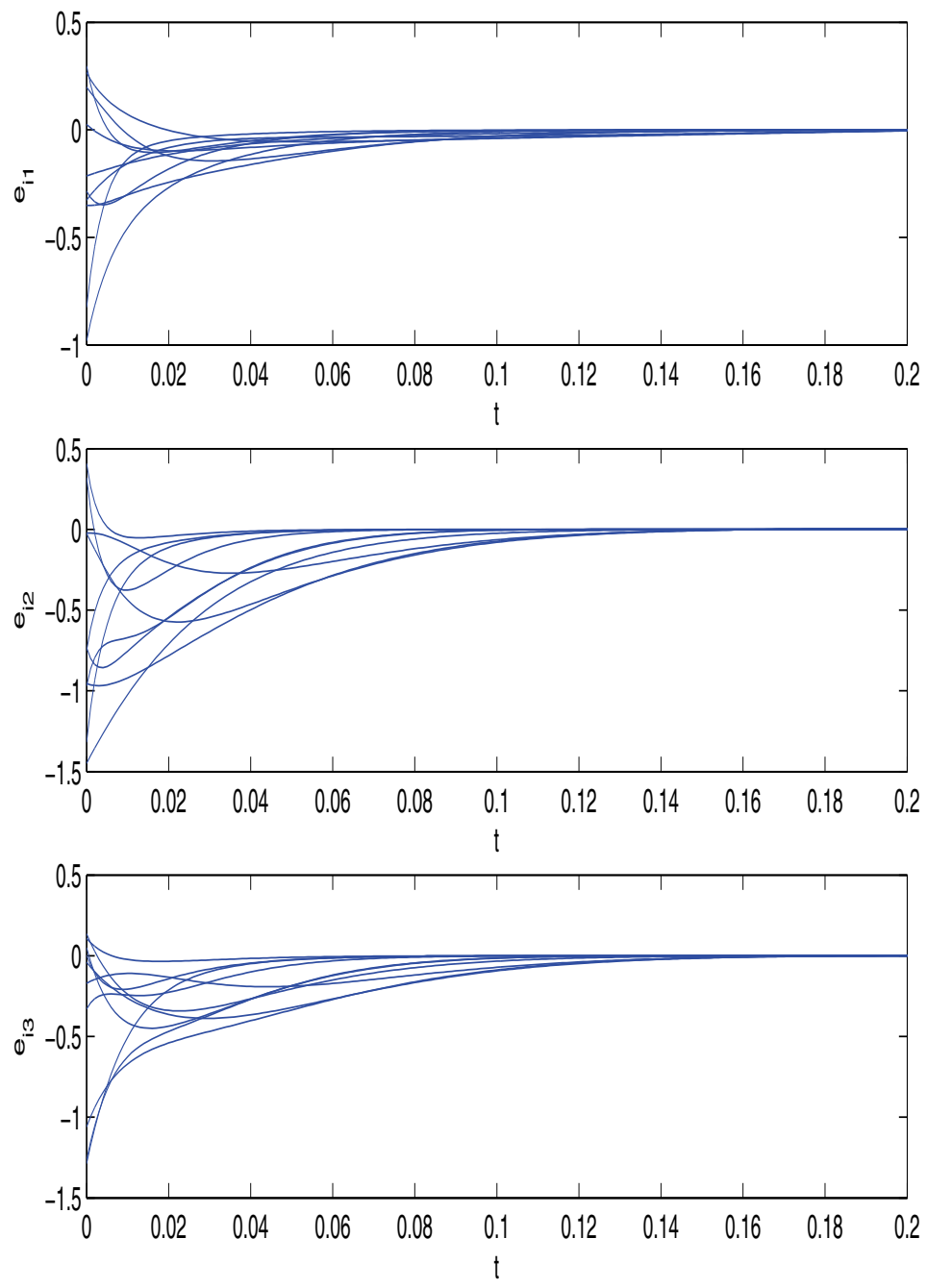

FIG. 6.2. Error states $e_{i}(t)$ of the complex network with regard to time $t$ in the example in subsection $6.1, i=1,2, \ldots, 11$. The whole network is globally synchronized as the error states asymptotically approach zero.

6.2. Synchronization via pinning control on a large-scale complex network. Choose $\Gamma=\operatorname{diag}(1,2,1), c=28, d_{i}=30$, and $N=100$. The simulation is performed on a scale-free complex network [3], where the direction for each edge is randomly chosen. The initial number of vertices is 5 , and in each time step, a new vertex is connected to 5 vertices with directed links according to preferential attachment. The designed directed scale-free network is disconnected into several strongly connected components. By using the algorithm in section 5 with the designed pinning schemes, a small fraction of all the vertices is controlled. The initial conditions are randomly chosen from $[-1,1]$ in each simulation, and synchronization can be reached in the whole network as shown in Figure 6.3, which is performed on 30 networks on average in each data. It is found that the percentage for the pinning controlled vertices becomes smaller as the network size becomes larger.

Copyright (c) by SIAM. Unauthorized reproduction of this article is prohibited. 


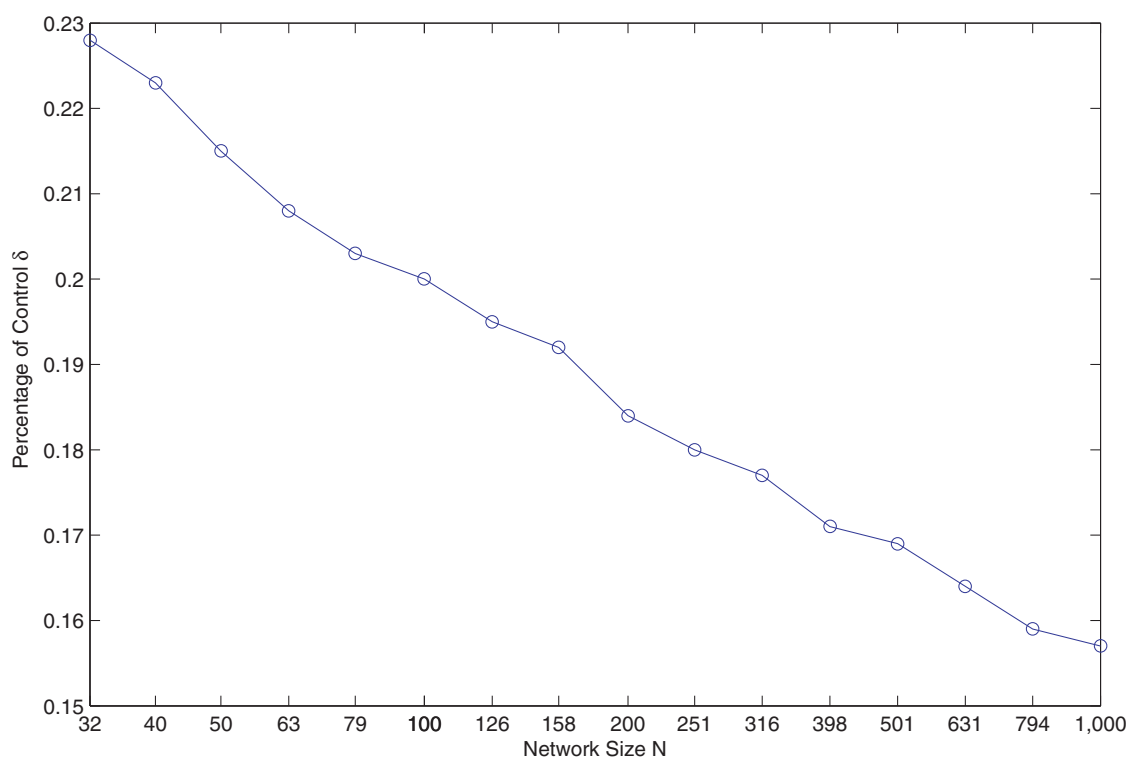

FIG. 6.3. Percentage $\delta$ of the controlled vertices of the complex network in the example in subsection 6.2 based on the network size $N$ with logarithmic axis.

7. Conclusions. In this paper, pinning synchronization of general complex dynamical networks, including strongly connected networks, networks with a directed spanning tree, weakly connected networks, and directed forests, has been discussed. A criterion for ensuring network synchronization on strongly connected networks was given. It was found that the vertices with very small in-degrees should be pinned first. In addition, it was shown that the original condition with controllers can be reformulated such that it does not depend on the form of the chosen controllers. Then, a criterion for achieving network synchronization on networks with a directed spanning tree, possibly composed of many strongly connected components, was derived. It was found that the components with very few connections from other components should be controlled, and the components with many connections from other components can achieve synchronization even without controls. Moreover, an effective pinning algorithm for reaching synchronization on a general complex dynamical network was proposed.

It is still a quite difficult problem to quantify at least how many vertices should be pinned and to determine what are the most important factors for a given complex network to realize synchronization. In addition, an involved complex network in nature is usually time-varying. Therefore, pinning schemes for synchronization in time-varying dynamical complex networks are more challenging nowadays. Moreover, it could be interesting to see how the proposed algorithm scales up as the number of vertices increases. Nevertheless, pinning synchronization on general complex dynamical networks is a very interesting topic with wide potential applications and deserves further investigation in the future.

\section{REFERENCES}

[1] A. Arenas, A. Diaz-Guilera, J. Kurths, Y. Moreno, and C. Zhou, Synchronization in complex networks, Physics Reports, 468 (2008), pp. 93-153. 
[2] S. Boccaletti, J. Kurths, G. Osipov, D. L. Valladares, and C. Zhou, The synchronization of chaotic systems, Physics Reports, 366 (2002), pp. 1-101.

[3] A. L. Barabási And R. AlBert, Emergence of scaling in random networks, Science, 286 (1999), pp. 509-512.

[4] S. Boyd, L. El Ghaoui, E. Feron, and V. Balakrishnan, Linear Matrix Inequalities in System and Control Theory, Stud. Appl. Numer. Math. 15, SIAM, Philadelphia, 1994.

[5] R. A. Brualdi and H. J. Ryser, Combinatorial Matrix Theory, Cambridge University Press, Cambridge, UK, 1991.

[6] Y. CaO, W. Yu, W. Ren, And G. Chen, An overview of recent progress in the study of distributed multi-agent coordination, IEEE Trans. Indust. Inform., 9 (2013), pp. 427-438.

[7] T. Chen, X. Liu, And W. Lu, Pinning complex networks by a single controller, IEEE Trans. Circuits Syst. I, 54 (2007), pp. 1317-1326.

[8] P. Delellis, M. di Bernardo, and F. Garofalo, Novel decentralized adaptive strategies for the synchronization of complex networks, Automatica J. IFAC, 45 (2009), pp. 1312-1318.

[9] M. di Bernardo, F. Garofalo, and F. Sorrentino, Effects of degree correlation on the synchronization of networks of oscillators, Int. J. Bifur. Chaos, 17 (2007), pp. 3499-3506.

[10] P. ERdös and A. RÉNYI, On random graphs, Pub. Math., 6 (1959), pp. 290-297.

[11] P. Erdös And A. RÉNYI, On the evolution of random graphs, Publ. Math. Inst. Hung. Acad. Sci., 5 (1960), pp. 17-61.

[12] R. O. Grigoriev, M. C. Cross, and H. G. Schuster, Pinning control of spatiotemporal chaos, Phys. Rev. Lett., 79 (1997), pp. 2795-2798.

[13] Y. Hong, G. Chen, And L. Bushnell, Distributed observers design for leader-following control of multi-agent networks, Automatica J. IFAC, 44 (2008), pp. 846-850.

[14] R. A. Horn and C. R. Johnson, Matrix Analysis, Cambridge University Press, Cambridge, UK, 1985.

[15] R. Li, Z. Duan, And G. Chen, Cost and effect of pinning control for network synchronization, Chinese Physics, 18 (2009), pp. 106-118.

[16] X. Li, X. WAng, And G. Chen, Pinning a complex dynamical network to its equilibrium, IEEE Trans. Circuits Syst. I, 51 (2004), pp. 2074-2087.

[17] W. Lu, Adaptive dynamical networks via neighborhood information: Synchronization and pinning control, Chaos, 17 (2007), 023122.

[18] W. Lu AND T. Chen, Synchronization of coupled connected neural networks with delays, IEEE Trans. Circuits Syst. I, 51 (2004), pp. 2491-2503.

[19] J. Lü AND G. Chen, A time-varying complex dynamical network models and its controlled synchronization criteria, IEEE Trans. Automat. Control, 50 (2005), pp. 841-846.

[20] H. LütKePohl, Handbook of Matrices, John Wiley, New York, 1996.

[21] L. Moreau, Stability of continuous-time distributed consensus algorithms, in Proceedings of the 43rd IEEE Conference on Decision and Control, IEEE Press, Piscataway, NJ, 2004, pp. 3998-4003.

[22] R. Olfati-Saber and R. M. Murray, Consensus problems in networks of agents with switching topology and time-delays, IEEE Trans. Automat. Control, 49 (2004), pp. 1520-1533.

[23] R. Olfati-Saber, Flocking for multi-agent dynamic systems: Algorithms and theory, IEEE Trans. Automat. Control, 51 (2006), pp. 401-420.

[24] L. M. Pecora and T. L. Carroll, Synchronization in chaotic systems, Phys. Rev. Lett., 64 (1990), pp. 821-824.

[25] M. Porfiri And M. Di Bernardo, Criteria for global pinning-controllability of complex networks, Automatica J. IFAC, 44 (2008), pp. 3100-3106.

[26] M. Porfiri And F. Fiorilli, Node-to-node pinning control of complex networks, Chaos, 19 (2009), 013122.

[27] W. Ren and E. AtKins, Distributed multi-vehicle coordinated control via local information exchange, Int. J. Robust Nonlinear Control, 17 (2007), pp. 1002-1033.

[28] C. W. Reynolds, Flocks, herds, and schools: A distributed behavior model, Computer Graphics, 21 (1987), pp. 25-34.

[29] F. Rum, B. W. Gordon, and M. Eghtesad, Consensus in Noisy Switching Directed Networks with Time-Delays, in Proceedings of the 2007 IEEE International Conference on Networking, Sensing, and Control, London, 2007, pp. 431-436.

[30] E. Semsar-Kazerooni and K. Khorasani, Switching control of a modified leader-follower team of agents under the leader and network topological changes, IET Control Theory Appl., 5 (2011), pp. 1369-1377.

[31] D. D. SilJak, Large-Scale Dynamic Systems: Stability and Structure, North-Holland, New York, 1979.

[32] Q. SONG AND J. CAO, On pinning synchronization of directed and undirected complex dynamical networks, IEEE Trans. Circuits Syst. I, 57 (2010), pp. 672-680.

Copyright $@$ by SIAM. Unauthorized reproduction of this article is prohibited. 
[33] F. Sorrentino, Effects of the network structural properties on its controllability, Chaos, 17 (2007), 033101.

[34] F. Sorrentino, M. di Bernardo, F. Garofalo, and G. Chen, Controllability of complex networks via pinning, Phys. Rev. E, 75 (2007), 046103.

[35] T. Vicsek, A. Cziok, E. B. Jacob, I. Cohen, and O. Shochet, Novel type of phase transition in a system of self-driven particles, Phys. Rev. Lett., 75 (1995), pp. 1226-1229.

[36] X. Wang And G. Chen, Synchronization in scale-free dynamical networks: Robustness and fragility, IEEE Trans. Circuits Syst. I, 49 (2002), pp. 54-62.

[37] X. Wang and G. Chen, Synchronization in small-world dynamical networks, Int. J. Bifur. Chaos, 12 (2002), pp. 187-192.

[38] X. Wang And G. Chen, Pinning control of scale-free dynamical networks, Phys. A, 310 (2002), pp. 521-531.

[39] D. J. Watts and S. H. Strogatz, Collective dynamics of 'small-world' networks, Nature, 393 (1998), pp. 440-442.

[40] C. Wu, Synchronization in arrays of coupled nonlinear systems with delay and nonreciprocal time-varying coupling, IEEE Trans. Circuits Syst. II, 52 (2005), pp. 282-286.

[41] C. WU, Synchronization in networks of nonlinear dynamical systems coupled via a directed graph, Nonlinearity, 18 (2005), pp. 1057-1064.

[42] C. Wu, Localization of effective pinning control in complex networks of dynamical systems, in Proceedings of the IEEE International Symposium on Circuits and Systems, IEEE Press, Piscataway, NJ, 2008, pp. 2530-2533.

[43] C. Wu, On the relationship between pinning control effectiveness and graph topology in complex networks of dynamical systems, Chaos, 18 (2008), 037103.

[44] C. Wu And L. O. ChuA, Synchronization in an array of linearly coupled dynamical systems, IEEE Trans. Circuits Syst. I, 42 (1995), pp. 430-447.

[45] J. Xiang and G. Chen, On the V-stability of complex dynamical networks, Automatica J. IFAC, 43 (2007), pp. 1049-1057.

[46] L. Xiang, Z. Liu, Z. Chen, F. Chen, and Z. Yuan, Pinning control of complex dynamical networks with general topology, Phys. A, 379 (2007), pp. 298-306.

[47] W. Yu, J. CaO, and G. Chen, Robust adaptive control of unknown modified Cohen-Grossberg neural networks with delay, IEEE Trans. Circuits Syst. II, 54 (2007), pp. 502-506.

[48] W. Yu, J. CAO, G. Chen, J. LÜ, J. Han, AND W. WeI, Local synchronization of a complex network model, IEEE Trans. Systems Man Cybernet. Part B, 39 (2009), pp. 230-241.

[49] W. YU, J. CAO, AND J. Lü, Global synchronization of linearly hybrid coupled networks with time-varying delay, SIAM J. Appl. Dyn. Syst., 7 (2008), pp. 108-133.

[50] W. Yu, G. Chen, And M. CAO, Distributed leader-follower flocking control for multi-agent dynamical systems with time-varying velocities, Systems Control Lett., 59 (2010), pp. 543552.

[51] W. Yu, G. Chen, And M. CAO, Some necessary and sufficient conditions for second-order consensus in multi-agent dynamical systems, Automatica J. IFAC, 6 (2010), pp. 10891095.

[52] W. Yu, G. Chen, And M. CaO, Consensus in directed networks of agents with nonlinear dynamics, IEEE Trans. Automat. Control, 56 (2011), pp. 1436-1441.

[53] W. Yu, G. Chen, M. CaO, and J. Kurths, Second-order consensus for multi-agent systems with directed topologies and nonlinear dynamics, IEEE Trans. Systems Man Cybernet. Part B, 40 (2010), pp. 881-891.

[54] W. YU, G. Chen, AND J. Lü, On pinning synchronization of complex dynamical networks, Automatica J. IFAC, 45 (2009), pp. 429-435.

[55] W. Yu, G. Chen, Z. WANG, AND W. YANG, Distributed consensus filtering in sensor networks, IEEE Trans. Systems Man Cybernet. Part B, 39 (2009), pp. 1568-1577.

[56] W. Yu, G. Chen, And J. Lü, Pinning control of general multi-agent systems, in Proceedings of the 9th World Congress on Intelligent Control and Automation, Beijing, China, 2012, pp. 1930-1935.

[57] W. Yu, J. Lü, G. Chen, Z. DuAn, And Q. Zhou, Estimating uncertain delayed genetic regulatory networks: An adaptive filtering approach, IEEE Trans. Automat. Control, 54 (2009), pp. 892-897.

[58] W. Yu, P. Delellis, G. Chen, M. Di Bernardo, and J. Kurths, Distributed adaptive control of synchronization in complex networks, IEEE Trans. Automat. Control, 57 (2012), pp. 2153-2158.

[59] W. Yu, W. Zheng, G. Chen, W. Ren, And J. CaO, Second-order consensus in multi-agent dynamical systems with sampled position data, Automatica J. IFAC, 47 (2011), pp. 14961503.

Copyright $@$ by SIAM. Unauthorized reproduction of this article is prohibited. 
[60] C. Zhou And J. Kurths, Dynamical weights and enhanced synchronization in adaptive complex networks, Phys. Rev. Lett., 96 (2006), 164102.

[61] J. Zhou, J. LU, AND J. LÜ, Adaptive synchronization of an uncertain complex dynamical network, IEEE Trans. Automat. Control, 51 (2006), pp. 652-656.

[62] J. Zhou, J. Lu, And J. Lü, Pinning adaptive synchronization of a general complex dynamical network, Automatica J. IFAC, 44 (2008), pp. 996-1003.

[63] J. Zhou, X. Wu, W. Yu, M. Small, and J. Lu, Pinning synchronization of delayed neural networks, Chaos, 18 (2008), 043111.

[64] Y. Chen, J. Lü, AND Z. Lin, Consensus of discrete-time multi-agent systems with transmission, Automatica J. IFAC, to appear.

Copyright (c) by SIAM. Unauthorized reproduction of this article is prohibited. 